\title{
Development, implementation, and evaluation of a novel guideline engine for pediatric patients with severe traumatic brain injury: a study protocol
}

\author{
Meagan R. Pilar ${ }^{1 *}$ (D) Enola K. Proctor ${ }^{1}$ and Jose A. Pineda ${ }^{2}$
}

\begin{abstract}
Background: Severe traumatic brain injury (TBI) is a leading cause of death and disability for children. The Brain Trauma Foundation released evidence-based guidelines, a series of recommendations regarding care for pediatric patients with severe TBI. Clinical evidence suggests that adoption of guideline-based care improves outcomes in patients with severe TBI. However, guideline implementation has not been systematic or consistent in clinical practice. There is also a lack of information about implementation strategies that are effective given the nature of severe TBI care and the complex environment in the intensive care unit (ICU). Novel technology-based strategies may be uniquely suited to the fast-paced, transdisciplinary care delivered in the ICU, but such strategies must be carefully developed and evaluated to prevent unintended consequences within the system of care. This challenge presents a unique opportunity for intervention to more appropriately implement guideline-based care for pediatric patients with severe TBI.
\end{abstract}

Methods: This mixed-method study will develop a novel technology-based bedside guideline engine (the implementation strategy) to facilitate uptake of evidence-based guidelines (the intervention) for management of severe TBI. Group model building and systems dynamics will inform the guideline engine design, and bedside functionality will be initially assessed through patient simulation. Using the Promoting Action on Research Implementation in Health Services (PARIHS) framework, we will determine the feasibility of incorporating the guideline engine in the ICU. Study participants will include pediatric patients with severe TBI and providers at three trauma centers. Quantitative data will include measures of guideline engine acceptance and organizational readiness for change. Qualitative data will include semi-structured interviews from clinicians. We will test the feasibility of incorporating the guideline engine in "real life practice" in preparation for a future clinical trial that will assess clinical and implementation outcomes, including feasibility, acceptability, and adoption of the guideline engine.

Discussion: This study will lead to the development and feasibility testing of an adaptable strategy for implementing guideline-based care for severe TBI, a strategy that meets the needs of individual critical care environments and patients. A future study will test the adaptability and impact of the bedside guideline engine in a randomized clinical trial.

Keywords: Head trauma, Traumatic brain injury, Children, Pediatrics, Implementation strategies

\footnotetext{
* Correspondence: Meagan.Pilar@wustl.edu

'Washington University in St. Louis, Brown School, One Brookings Drive,

Campus Box 1196, St. Louis, MO 63130, USA

Full list of author information is available at the end of the article
}

(c) The Author(s). 2020 Open Access This article is distributed under the terms of the Creative Commons Attribution 4.0 International License (http://creativecommons.org/licenses/by/4.0/), which permits unrestricted use, distribution, and reproduction in any medium, provided you give appropriate credit to the original author(s) and the source, provide a link to the Creative Commons license, and indicate if changes were made. The Creative Commons Public Domain Dedication waiver (http://creativecommons.org/publicdomain/zero/1.0/) applies to the data made available in this article, unless otherwise stated. 


\section{Contributions to the literature}

- Research has shown that the adoption of guideline-based care improves outcomes for patients with severe TBI. However, these guidelines have not been applied systematically in the intensive care unit clinical setting

- Technology-based implementation strategies may be uniquely suited to the fast-paced and transdisciplinary approaches of the intensive care unit. Technology-based strategies may facilitate the timely, consistent, and sustained application of evidence-based care in routine practice.

- This study combines implementation science frameworks and systems science to develop an innovative implementation strategy, the bedside guideline engine. Our goal is to reduce the gap between evidence-based knowledge and actionable patient data to ultimately impact patient outcomes.

\section{Background}

\section{Prevalence and treatment of TBI in children}

An estimated 1.7 million Americans sustain severe traumatic brain injury (sTBI) annually, resulting in more than 50,000 deaths [1]. The majority of sTBI survivors must contend with considerable disabilities and financial burden [2]. In 2013, more than 640,000 children ages 0-14 visited the emergency department due to TBI, resulting in nearly 18,000 hospitalizations [3]. Ultimately, more than 2100 children die annually because of sTBI [4]. Young children are particularly at-risk due to falls, as well as motor vehicle accidents and assault $[4,5]$. Not surprisingly, sTBI is a leading cause of death and disability in children in the USA [6], making it a significant public health problem.

In 2019 the Brain Trauma Foundation (BTF) released a third edition of guidelines for the acute medical management of sTBI in infants, children, and adolescents [7]. These recommendations center around the avoidance of secondary insults to the injured brain. Secondary insults are abnormal physiological states (e.g., increased intracranial hypertension, low blood pressure, and fever) that aggravate the primary injury (i.e., injury the brain sustains at the time of trauma). Secondary insults have been consistently associated with poor outcomes after sTBI. Consequently, the BTF guidelines provide evidence-based recommendations and best practices regarding treatment interventions and patient monitoring [7]. Multiple reports provide evidence that the adoption of guideline-based care improves mortality and functional outcome in patients with sTBI [8-13]. The Centers for Disease Control and Prevention studied the impact of the BTF guidelines and found that the implementation and widespread use could decrease deaths by $50 \%$ and result in economic savings for both sTBI patients (estimated $\$ 288$ million) and society as a whole (estimated $\$ 3.8$ billion) [14].

\section{Challenges for promoting TBI guidelines in practice}

Despite the availability of evidence-based approaches for treating TBI, the implementation of guideline-based recommendations is limited, particularly in pediatric patients [9-11, 13, 15-19]. The guidelines have not been systematically applied in clinical practice, and compromised fidelity to guideline-based care results in large variability in care and outcomes [20, 21]. A growing body of literature describes the difficulties associated with implementation $[22,23]$. For example, individual-level characteristics (e.g., physicians' knowledge of current recommendations), external conditions (e.g., insufficient time or resources to implement guideline), and patient preferences against a guideline can each pose barriers for care [24-29].

Additional challenges for widespread use of guidelinebased care include a lack of strategies that account for the complex, multilevel nature of sTBI care and medical staff [30, 31]. Medical teams caring for children with sTBI are comprised of providers with different levels of expertise, diverse backgrounds and, in many instances, limited experience working together as a cohesive team. This revolving team dynamic creates fragmented communication and less than optimal working conditions for shared decision-making across disciplines [32].

The dissemination of sTBI-related materials also challenges guideline-based pediatric critical care practice. Research has highlighted the difficulty for physicians in remaining up-to-date with current recommended practices and guidelines [33]. Unfortunately, deployment methods for guidelines typically include disseminating paper or electronic materials, which are either distributed to individual team members or available through a shared document archive. These dissemination methods are problematic, particularly in the intensive care environment because the resources are often not readily available when team members are communicating or making time-sensitive decisions at the bedside. The lack of effective dissemination and implementation strategies to scale up and sustain guideline-based care for children with sTBI represents an opportunity to improve care and outcomes. These strategies need to be tailored to individual patients and the unique environment in any given intensive care setting.

\section{Technology-based strategies in clinical care}

Novel technology-based strategies may be uniquely suited to the fast-paced and transdisciplinary approaches of a clinical care setting like the intensive care unit (ICU). A recent summary by the National Cancer Institute emphasized the efficacy of technology-based strategies to improve care [34]. However, such technology- 
based strategies must be carefully developed and evaluated to prevent unintended consequences within the system of care [10, 11, 22]. Given such complexities, effective and sustained application of evidence-based guidelines will require newly designed, breakthrough implementation strategies that are applicable to real world ICU practice [10, 33, 35]. This challenge presents a unique opportunity for intervention to more appropriately disseminate and implement guideline-based care for pediatric patients with sTBI.

\section{Innovative implementation strategy for sTBI patients}

Building on existing knowledge of pediatric sTBI, the proposed study will harness implementation and system dynamics sciences to inform the development of a patient-centered strategy to accelerate the adoption and sustained delivery of care based on the best available evidence. This study will develop and test the feasibility of a novel clinical implementation strategy-hereafter called the bedside guideline engine (BGE) - to facilitate timely and consistent delivery of evidence-based sTBI care. Table 1 summarizes current challenges for analyzing patient data, communicating between care team members, and making bedside time-sensitive decisions, while highlighting the advantages of the BGE [36-38].

This technology-based implementation strategy will bolster consistent clinical decision-making by providing efficient access to actionable patient information, as well as guideline-based therapeutic options. The BGE will consist of (1) the Component Neuromonitoring System (CNS Monitor, Moberg Research, Ambler, PA), an FDA approved bedside device that displays relevant patient information and records therapeutic interventions and (2) a computer-readable clinical pathway based on the pediatric BTF sTBI guidelines [19]. It will incorporate features of implementation strategies associated positive impact on both clinical practice and patient outcomes, including point of care decision support and real time automatic provision of recommendations-rather than just clinical assessments [36, 37, 39]. The BGE will also allow workflow tracking and systematic evaluations of team behavior to better understand the factors that facilitate or disable sustainable implementation of evidence-based care. Ultimately, the BGE will empower providers with consistent knowledge of evidence-based recommendations, which will facilitate more reliable and expedited care. Importantly, the BGE will allow for adaptation of evidence-based recommendations to individual patient needs and clinical scenarios.

\section{Conceptual framework}

The proposed research activities are based on the Promoting Action on Research Implementation in Health Services (PARIHS) framework, which describes how three key interacting elements-evidence, context, and facilitation-influence the successful implementation of evidence-based practices [40, 41]. Table 2 outlines how the PARIHS framework will inform the design and implementation of the BGE in the ICU.

\section{Methods}

The focus of this project is to develop and test the feasibility of a novel technology-based BGE to facilitate timely adherence to evidence-based guidelines and bolster consistent clinical decision-making. The team will develop and evaluate a technology-driven implementation strategy (the BGE) that facilitates the uptake of evidence-based guidelines for management of sTBI at the bedside (aim 1). We will then determine the feasibility of incorporating the BGE in the ICU environment

Table 1 Advantages of BGE over current approaches for delivery of guideline-based sTBI care

\begin{tabular}{|c|c|c|}
\hline Issue & Current Approach & BGE \\
\hline $\begin{array}{l}\text { Inefficient access to guideline content } \\
\text { delays care }\end{array}$ & $\begin{array}{l}\text { Requires accessing paper or electronic depository } \\
\text { documents (time-consuming, inconsistent, and } \\
\text { difficult) }\end{array}$ & $\begin{array}{l}\text { Content is always present and highly visible on } \\
\text { dedicated bedside device for immediate review }\end{array}$ \\
\hline $\begin{array}{l}\text { Indirect or absent link between content } \\
\text { and patient data interferes with fidelity } \\
\text { to guidelines }\end{array}$ & $\begin{array}{l}\text { Linking content to patient data requires active effort } \\
\text { by providers through multi-step processes }\end{array}$ & $\begin{array}{l}\text { Guideline content is automatically linked to the } \\
\text { patient's momentary condition }\end{array}$ \\
\hline $\begin{array}{l}\text { Patient's condition triggers alerts, but } \\
\text { alerts are not linked to recommended } \\
\text { care }\end{array}$ & $\begin{array}{l}\text { Static guidelines: providers must identify alerts and } \\
\text { link them to recommended care }\end{array}$ & $\begin{array}{l}\text { Alerts automatically trigger content-based recom- } \\
\text { mendations, which are displayed at the bedside } \\
\text { alongside patient data }\end{array}$ \\
\hline Information overload & $\begin{array}{l}\text { - Care team members individually merge data from } \\
\text { bedside monitor, medical record, and guideline } \\
\text { content. } \\
\text { - Access to patient physiology trends is often } \\
\text { difficult or not available. }\end{array}$ & $\begin{array}{l}\text { - Data from relevant sources is merged and } \\
\text { automatically linked to guideline-based content. } \\
\text { - High-resolution trends of patient physiology are } \\
\text { available to facilitate data review and decision- } \\
\text { making }\end{array}$ \\
\hline $\begin{array}{l}\text { No efficient method for ongoing } \\
\text { evaluation of timeliness of therapy and } \\
\text { fidelity to guidelines }\end{array}$ & $\begin{array}{l}\text { - Clinical care is recorded in the medical record, } \\
\text { which requires retrospective data collection } \\
\text { (manual or semi-automated). } \\
\text { - Timeliness of therapy is just an estimate. }\end{array}$ & $\begin{array}{l}\text { - Automated data extraction for efficient audits of } \\
\text { care } \\
\text { - Tracks time-stamped team interventions and } \\
\text { feedback }\end{array}$ \\
\hline
\end{tabular}


Table 2 Timeline for designing and evaluating the BGE while including core element of the PARIHS framework

\begin{tabular}{|c|c|c|c|}
\hline \multicolumn{3}{|l|}{ Year 1} & \multirow{2}{*}{$\begin{array}{l}\text { Years 1-2 } \\
\text { Facilitation }\end{array}$} \\
\hline $\begin{array}{l}\text { PARIHS } \\
\text { core elements }\end{array}$ & Evidence & Context & \\
\hline Approach & $\begin{array}{l}\text { BTF guidelines for the acute medical } \\
\text { management of severe TBI in infants, } \\
\text { children, and adolescents }\end{array}$ & $\begin{array}{l}\text { Assessment of the } \\
\text { ICU environment and } \\
\text { culture }\end{array}$ & $\begin{array}{l}\text { 1. Incorporation of the BTF guideline content into a } \\
\text { computerized pathway } \\
\text { 2. Linking the pathway to patient data (completes the BGE) } \\
\text { 3. Evaluation of BGE initial acceptance, perceived benefit, } \\
\text { challenges to use, and adoption potential (informs BGE } \\
\text { design and adaptation) } \\
\text { 4. Feasibility testing in simulation environment and real world } \\
\text { practice (informs refinement of the BGE and design of future } \\
\text { clinical trial) }\end{array}$ \\
\hline \multicolumn{3}{|c|}{ Baseline bedside practice data collection, contextual evaluation } & Evaluation of unadapted and adapted technology \\
\hline
\end{tabular}

(aim 2). This will include evaluation of the extent of adaptation of the BGE that is required at each of three participating ICUs. Feasibility testing will yield real world practice identification of site-specific barriers and facilitators to implementation of the BGE. More information for each step in will be described in the following section.

\section{Aim 1: Develop and evaluate a technology-driven implementation strategy (the BGE) that facilitates the uptake of evidence-based guidelines for management of sTBI at the bedside \\ Study population, subjects, and recruitment}

In aim 1, we will develop a novel technology-based bedside BGE that will make guideline-based care easier for providers to access and implement in real time. Development will take place at three regional pediatric trauma centers, which provide care for the majority of sTBI patients in the state of Missouri. The BGE will be developed using an interdisciplinary team of providers and researchers who have experience in implementation and system dynamics science, clinical decision support tools, critical care, neurosurgery, and sTBI.

\section{Procedures, instruments, and design}

Capturing the ICU environment and culture Staff observations and interviews

To inform the development of the BGE, we will first capture the ICU environment and culture from each clinical site. Using staff interviews and ethnographic observations [42], we will describe organizational patterns (leadership, team composition, workflow) in participating ICUs.

\section{Participatory group modeling}

We will then use group model building and systems dynamics to inform the design of the BGE. This process will shift information gathering to a structured approach instead of more traditional focus group approaches [43]. Participatory group model building methods are based on community-based system dynamics (CBSD), a participatory method for involving communities (in this case health care providers) in the process of understanding and changing systems from the endogenous or feedback perspective of system dynamics [44]. The method will provide a visual representation of relationships that impact the BGE's flow with input from physician leaders, nursing leaders, and bedside care providers.

Members of the research team will travel to all clinical sites to build knowledge around hospital functioning in each ICU (leadership, team composition, workflow for decision-making). The research team will convene multidisciplinary care teams at each collaborating site to conduct group model building workshops. This form of system dynamics method incorporates stakeholder group participation in modeling the system to fully understand its complexities $[45,46]$. The workshop outputs will include causal models that outline barriers and feedback loops, which will enable visual representation of relationships and mechanisms important to successful uptake of the BGE [47]. The insights from this work will inform the BGE's workflow design by delineating each ICU's initial inertia and efficiency-factors that impact how the BGE will benefit local workflow [48-50].

Designing the implementation strategy: BGE development The BGE will serve as an implementation strategy to enhance the adoption and sustained delivery of evidence-based clinical care for sTBI patients. It will be developed using the InfoMap Markup Language (IML), a novel tool that allows development of a computer-readable clinical pathway from a generic guideline. To reduce information overload and optimize alignment with clinical practice, BTF guideline IML content will be organized via Learning Management Systems principles [51]. The IML pathway will then be deployed into a dedicated personal computer using CarePath, a recently developed software platform that reads IML content and generates a graphic flowchart. The personal computer will be mounted next to the bedside 
Component Neuromonitoring System (CNS) monitor (Moberg Research, Ambler, PA), an FDA-approved multimodality bedside monitor for patients with brain injury. Moberg Research developed this technology with funding from the National Institutes of Health (National Institute of Nursing Research) and the Department of Defense.

With technical support from Moberg Research, we will adapt the IML technology to fit aspects of sTBI pathophysiology and treatment that are unique to the pediatric population. The Moberg CNS monitor allows high-resolution $(0.56 \mathrm{~Hz})$ prospective digital archival of vital signs that measure exposure to secondary insults. This software will generate a graphic flowchart and connects the BGE to patient physiology, nursing input, and instructional content while recording time-stamped nursing and physician interventions.

All output will be displayed on the CNS monitor, which continuously collects and displays the patient's vital sign trends, including input from neuromonitoring devices (i.e., intracranial pressure monitor). By directly downloading vital signs from the bedside CNS monitor, the BGE will provide high-resolution trends instead of low-resolution data from hourly documentation in the medical record. The display will give clinicians real-time bedside access to patient assessments, thus facilitating access to more accurate data regarding injury progression and response to therapy [30]. Additionally, the display will provide recommendations for clinicians with the next step suggested by the patient's pathophysiology, nursing input, and clinical knowledge from the BTF guidelines.
During the development of the BGE, we will use an adaptation of the Stages of Implementation Completion tool (SIC) to monitor and evaluate the completion of implementation activities, the length of time taken, and the proportion of activities completed [52]. Stages to be tracked are (1) incorporation of critical elements of the ICU environment and culture into the BGE design, (2) incorporation of the BTF guidelines into the CarePath pathway, and (3) linking of patient data and CarePath using the CNS monitor [52].

It is important to note that the CNS monitor is not meant to substitute the clinician in the decision-making process. Instead, it is a tool for clinicians that is linked to patient pathophysiology and translates evidence-based guidelines into an electronic, interactive form available at the bedside. The BGE will track clinician-patient interactions and the patient's response-or lack of response-to those interactions. Figure 1 provides an example of the BGE incorporated into the CNS monitor.

Evaluating the BGE's initial acceptability After the BGE has been designed, we will present it to ICU team members from all three sites and gauge initial BGE acceptance. Finally, we will use focus groups with ICU team members to assess acceptability and suggested improvements for the BGE [53]. These measures and processes will be further explained below.

\section{Measures}

Technology Acceptance Evaluation Model

We will evaluate acceptance of the technology-based BGE using a standardized technology acceptance

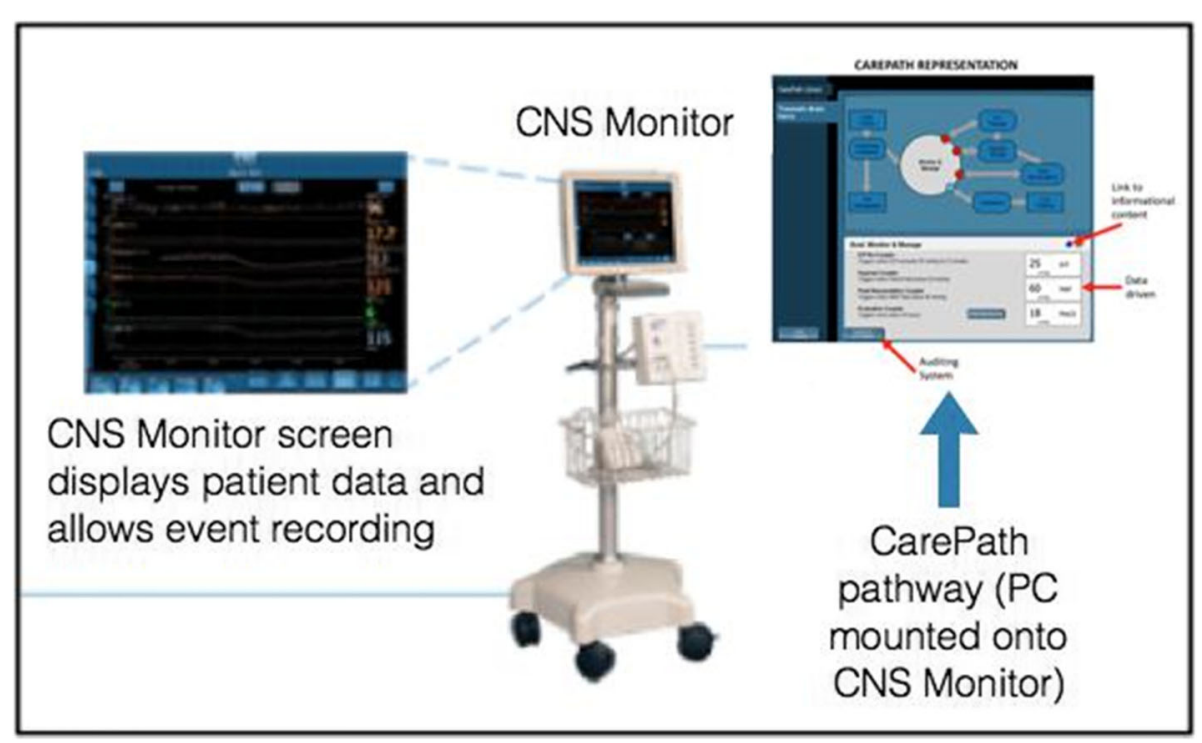

Fig. 1 Example of CNS monitor display 
questionnaire [54]. The questionnaire measures perceived ease of use, usefulness, and intention to use with seven-point Likert scales for all items.

Perceived Attributes of eHealth Innovations Questionnaire We will evaluate the BGE's acceptability using a 30item questionnaire with demonstrated validity and reliability [55]. This measure will explore attributes of the BGE that may predict bedside adoption: relative advantage (better than existing solutions), compatibility (comports with adopters' existing values and experiences), complexity/simplicity (relative difficulty or simplicity of use), trialability (can be used experimentally), and observability (results visible to outsiders). ICU team members using the BGE in their respective roles will complete a survey after training but before implementation in the patient simulation environment (time point 1).

\section{Data collection and data analysis}

\section{Capturing the ICU environment and culture Staff} observations and interviews

Additionally, acceptance of the innovative BGE will be gauged by a collection of qualitative input from users $[56,57]$. Data will be collected in separate sessions at each of the three clinical sites and will be analyzed using the grounded theory qualitative method [58].

Evaluating the BGE's initial acceptability Technology Acceptance Evaluation Model

All quantitative data will be managed and analyzed using the $\mathrm{R}$ statistical computation system [59]. As the questionnaire measures-perceived ease of use, efficacy, and intention to use/trust-have a skewed distribution, we specify an ordered logistic model [10]. The dataset also has a multiply-aggregated structure, as participants are nested within their group's session, and sessions are nested within clinical sites. To address the violation of independent-observation assumption, we apply a three-level mixed model to the data analysis as follows:

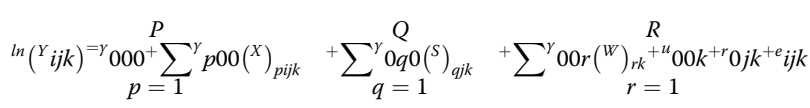

where $\ln \left(Y_{i j k}\right)$ is the outcome variable of interest for the $i$ th participant working in the $j$ th session from the $k$ th site, $(X)_{p i j k}$ are $P$ participant-level variables, $(S)_{q j k}$ are $Q$ session-level variables, $(W)_{r k}$ are $R$ site-level variables, $u_{0 o k}$ is a random effect for the $k^{\text {th }}$ site, $r_{O j k}$ is a random effect for the $j$ th session conducted in the $k$ th site, and $e_{i j k}$ is a residual term. Many individual-, session-, and site-level characteristics are coded as dichotomous variables. As such, the exponent of estimated coefficient $\exp (\gamma)$ for a dichotomous variable indicates percentage difference between groups on the level of technology acceptance. These effects, in conjunction with statistical significance testing, help discern barriers and facilitators of the implementation of BGE, which will be incorporated into the BGE's design to optimize functionality and acceptance.

Perceived Attributes of eHealth Innovations Questionnaire

Responses will be aggregated to produce an overall assessment of the five criteria for design and adjustment purposes. Given the environment's highly involved healthcare providers, we expect a high response rate and low missing data (e.g., refusals, "Do not Know" answers). Responses will be used to assess validity and reliability via confirmatory factor analysis on the five criteria, with exploratory factor analysis used to validate the contribution of individual questions. Table 4 in Atkinson 2007 [55] provides a general template for mapping specific questions to criteria. Cronbach's alpha will test for internal consistency. The outcome will be environmental, ergonomic, and medical workflow information for design refinement.

Focus groups

Focus groups will include 5-8 team members of the same role (i.e., nurses, respiratory therapists). We will use principles of the Contextualized Technology Adaptation Process (CTAP) to guide the focus groups, as this framework considers multilevel influences on technology use and is specifically intended for technologies that are in the development or adaptation process [60]. In line with the CTAP, we will focus on the core domains of functionality (i.e., the range of operations provided by the technology) and presentation (i.e., the style of communicating information to the user), which provide a general structure for our sample stimulus questions.

These focus groups will allow users who have previously trialed the BGE to identify useful, irrelevant, and problematic features to inform refinement efforts [60]. Using a primarily deductive approach, we will conduct a directed content analysis by predetermining theoretically driven categories, coding themes based on those categories, and then forming new categories for responses to which the initial coding scheme could not be applied [61]. We will employ a QUAN $\rightarrow$ qual sequential triangulation, using qualitative feedback to explain and expand upon the quantitative findings derived from the questionnaire data captured from the standardized technology acceptance and perceived attributes of eHealth innovations questionnaires $[54,55]$. The knowledge gained will inform adaptation of the BGE prior to evaluation in the patient simulation environment.

Aim 2: Determine the feasibility of incorporating the BGE in the ICU environment

\section{Study population, subjects, and recruitment}

In aim 2, we will test the feasibility of incorporating the BGE into clinical practice. We will prospectively enroll 
36 pediatric patients with sTBI in the ICU from the three proposed trauma centers (approximately 12 patients per clinical site) during the 24-month period. This sample size was selected based on feasibility and practical considerations, including the number of patients typically diagnosed with sTBI. Pediatric patients with sTBI (measured as a Glasgow Coma Scale score of $\leq 8$ ) admitted to the ICU at each clinical site will be screened for participation. Inclusion criteria for pediatric patients are as follows: (1) age 0-17 years, (2) accidental or nonaccidental sTBI, and (3) placement of an intracranial monitoring device for clinical care. In the final stages of feasibility testing, written informed consent from the patient's parents or legal guardian will be obtained by the research team before testing of the BGE, and safety measures will be in place to assure testing of the BGE does not compromise patient care. Given the low potential risk to human subjects and the challenges encountered when seeking written informed consent shortly after children are admitted to the ICU with sTBI, we will request the option of delayed written informed consent (up to $48 \mathrm{~h}$ ) for cases when delaying consent is necessary (e.g., if parents are not reasonably available at the time of admission). For patients who choose not to participate in our feasibility testing, consent for prospective data collection from the medical record will be obtained. The data will be used for initial exploration of penetration of the BGE into clinical practice.

\section{Procedures, instruments, and design}

Feasibility testing: patient simulation Once the BGE has been developed, we will introduce the BGE to a multidisciplinary group of ICU clinicians representing all clinical sites. We will use an adaptation of a previously described simulation module for trauma and critical care patients [62] to conduct the patient simulation using resources from the Sigh Pediatric Simulation Center at St. Louis Children's Hospital, St. Louis, MO. We will then reapply the Perceived Attributes of eHealth Innovations questionnaire and the Technology Acceptance Evaluation Model (time point 2) and capture qualitative user input regarding the BGE in the simulation environment. This approach will inform evaluation and adjustment of the BGE and will assist with final configuration prior to bedside feasibility testing at the clinical sites.

Feasibility testing: clinical implementation

After being tested in a patient simulation environment and revised accordingly, we will conduct feasibility testing in our three clinical sites. Clinical implementation will be bolstered using an implementation facilitator (IF), as well as trainings for clinical staff.

Implementation facilitator
Feasibility testing at each clinical site will be conducted with assistance from a Pediatric Neurocritical Care Program implementation facilitator (IF). The IF will assure adequate dissemination of the knowledge needed for users to understand and use the tool. This will occur through the collaboration with local multidisciplinary stakeholders (i.e., each ICU's physician and nursing leadership and bedside care providers) and tangible implementation goals with respect to uptake and fidelity. The goals include use of the BGE for bedside decision-making, including incorporation of evidencebased care recommendations. The IF will also lead the team as they set goals and work through the stages of the innovation decision process [63]. The incorporation of an IF has previously used when implementing new protocol and technologies [64]; however, it is still uncommon in acute care settings like the ICU. Providing expertise on implementation and dissemination will foster success and ease of adoption.

Training

Training will be provided to all users with initial emphasis on a subset of clinicians (charge nurses and ICU attendings), assuring a trained user will be available at all times to coach clinicians during the bedside feasibility testing. The IF, knowledgeable in the context and workflow of the participating ICUs, will work closely with the Washington University Dissemination and Implementation Research Core to apply a training program using a template developed by the State Implementation and Scaling-up of Evidence-based Practices (SISEP) Center at the University of North Carolina [65]. This approach emphasizes training and coaching on new skills that are necessary for effective use of innovations. Training will include videos developed in collaboration with Moberg ICU Solutions. The education process will continue until all providers are trained on BGE use, providing coaching, or advising and assisting through completion of the evaluation, to capture immediate feedback and increase fidelity [63, 64, 66, 67].

Clinician interviews

Finally, we will evaluate the BGE's practicality (predicted cost, burden and benefit to ICU team), integration (extent of use and impact on other activities in the ICU), and expansion (perception of how expanded deployment would work) [68]. Information will be obtained through semi-structured interviews of clinicians (nurses, respiratory therapists, attending physicians and physicians in training) and hospital administrators $[67,69]$.

\section{Data collection and data analysis}

Feasibility testing: patient simulation Perceived Attributes of eHealth Innovations Questionnaire 
Data extracted will be analyzed using the same methodology as aim 1 .

Cognitive interviewing

Use a think-aloud cognitive interviewing to obtain in vivo thoughts and reactions in the simulation environment [70].

Feasibility testing: clinical implementation During the feasibility testing phase, the Technology Acceptance Evaluation Model and the Perceived Attributes of eHealth Innovations questionnaire will be distributed again [54, 55]. Similarly, cognitive interviewing will be used after implementation. Quantitative and qualitative data will be analyzed using the same methodology as previously described.

\section{Trial status}

Study procedures have been approved by the Institutional Review Board at Washington University in St. Louis and the University of Missouri, Kansas City. Data collection and BGE designing are ongoing at the time of submission of this manuscript (July 2019).

\section{Discussion}

To improve clinical outcomes for children with sTBI, the best available evidence-based care must be more effectively tested and consistently applied in real-world practice. This study will develop and evaluate an innovative implementation strategy (a technology-based BGE) to facilitate timely delivery of evidence-based care of children with sTBI. The overall objective is to reduce fragmentation between evidence-based guideline information and actionable patient data, a barrier that typically stands in the way of fidelity to guidelines. If successful, this approach will also prove useful for implementation of guidelines for treatment of other complex and time sensitive conditions in the ICU environment.

Our innovative approach will incorporate state of the art technology and input from real world practice at three pediatric trauma centers. Our approach proposes significant change in the way care is delivered to children with sTBI by making evidence-based care easier for providers to apply in a timely fashion. The BGE will incorporate real-time feedback from the patient's physiology through the CNS monitor while giving providers access to high resolution data trends; the $\mathrm{BGE}$ will then link these data to an evidence-based guideline with the goal of improving decision-making and timely delivery of therapy. The BGE will be built with capacity to efficiently incorporate new evidence as it becomes available. Ultimately, we anticipate this strategy will create a sustainable approach to efficient and consistent care for critically ill children with sTBI.

If successful, this approach may prove useful for the implementation of evidence-based guidelines in other settings where time sensitive, complex care is provided (e.g., other ICU conditions, the emergency department, or battlefield).
Other ICU conditions that may benefit from this approach include management of nutrition, sedation/analgesia administration, treatment of life-threatening infections, and prevention of hospital acquired conditions. The innovation developed in this project may also contribute to the design of a future model of care that can adapt to different institutions and have a sustained impact on outcomes.

\section{Limitations}

There are limitations to this project that should be noted. First, though previous and emerging evidence supports the effectiveness of guideline-based care in adults and children with sTBI, better understanding of the pathophysiology and effectiveness of specific interventions is needed. Additionally, in this 2-year project, it is possible that the number of patients in the feasibility testing phase will not be sufficient to adequately test the BGE. The participating adult trauma center ICU may have access to fewer pediatric patients, but since pediatric trauma patients can receive care at adult trauma centers, we believe it is important to gain knowledge from our feasibility testing in a non-pediatric trauma center/ICU environment. Given that we are using process-of-care outcomes as our metrics, we anticipate patient accrual will not be a significant limitation. Future studies are being planned to test the impact of the BGE on patient outcomes.

\section{Conclusions}

Previous research has highlighted the need for an innovative implementation strategy to scale up and sustain guideline-based care. As such, we hope to use the BGE to improve outcomes in children with sTBI by facilitating timely, consistent, and sustained application of evidence-based care in routine practice. Our long-term goal is to develop and rigorously test an implementation strategy that fits the realities of clinical practice and individual patients and contributes to sustained implementation of guideline-based care for children with sTBI. This innovative proposal enables us to take the first step in this long-range implementation program.

Development of the BGE meets the broad need to decrease unnecessary variation in care and improve compliance with desired therapies, highlighting the relevance and generalizability of our approach to the wider ICU population [71]. We believe the proposed BGE will improve the application of adaptable guideline-based TBI care to meet the needs of individual ICU environments and patients. Additionally, this implementation strategy might guide the successful adaptation of our approach at other institutions. In a future comparative effectiveness clinical trial, we will propose scaled implementation of the BGE anticipating this approach will be superior to traditional care in translating knowledge into practice, positively impacting outcomes for children with sTBI. 


\section{Abbreviations}

BGE: Bedside guideline engine; BTF: Brain Trauma Foundation; CBSD: Community-based system dynamics; CNS: Component Neuromonitoring System; CTAP: Contextualized Technology Adaptation Process; ICU: Intensive care unit; IF: Implementation facilitator; IML: InfoMap Markup Language; PARIHS: Promoting Action on Research Implementation in Health Services Framework; SIC: Stages of Implementation Completion; SISEP: State Implementation and Scaling-up of Evidence-based Practices; sTBI: Severe traumatic brain injury; TBI: Traumatic brain injury

\section{Authors' contributions}

JP led the conception and design of the study. MP wrote the manuscript. EP revised the manuscript carefully for important intellectual content. All authors read and approved the final manuscript.

\section{Funding}

The study was funded by grants \# R21HD086784-01 (National Institute of Child Health and Human Development) and DR-2017-358a (St. Louis Children's Hospital Foundation). The funder had no role in the study design, the writing of the manuscript, or the decision to submit this manuscript for publication.

\section{Availability of data and materials}

Data sharing is not applicable to this article as no datasets were generated or analyzed during the current study.

\section{Ethics approval and consent to participate}

Approval to conduct the study was gained from the Institutional Review Board at Washington University in St. Louis and the University of Missouri, Kansas City (IRB ID \# 201602153).

\section{Consent for publication}

Not applicable

\section{Competing interests}

The authors declare that they have no competing interests.

\section{Author details}

'Washington University in St. Louis, Brown School, One Brookings Drive Campus Box 1196, St. Louis, MO 63130, USA. ${ }^{2}$ Children's Hospital Los Angeles/University of Southern California, Keck School of Medicine, 4650 Sunset Blvd, Los Angeles, CA 90027, USA.

Received: 3 January 2020 Accepted: 13 January 2020

Published online: 25 February 2020

\section{References}

1. Coronado VG, Xu L, Basavaraju SV, et al. Surveillance for traumatic brain injury-related deaths - United States, 1997-2007. MMWR Surveill Summ. 2011;60(5):1-32 Available from: https://www.ncbi.nlm.nih.gov/ pubmed/21544045.

2. Dash HH, Chavali S. Management of traumatic brain injury patients. Korean J Anesthesiol. 2018:71(1):12-21 Available from: https://www.ncbi.nlm.nih. gov/pmc/articles/PMC5809702/.

3. Taylor CA, Bell JM, Breiding MJ, Xu L. Traumatic brain injury-related emergency department visits, hospitalizations, and deaths-United States, 2007 and 2013. MMWR Surveill Summ. 2017;66 Available from: https://www. cdc.gov/mmwr/volumes/66/ss/ss6609a1.htm. Accessed 1 Dec 2019.

4. Langlois JA, Rutland-Brown W, Thomas KE: Traumatic brain injury in the United States: Emergency department visits, hospitalizations, and deaths. Atlanta, GA: Centers for Disease Control and Prevention, National Center for Injury Prevention and Control, 2006 Available from: https://www.cdc.gov/ traumaticbraininjury/tbi_ed.html

5. Centers for Disease Control and Prevention. Rates of TBI-related emergency department visits by age group — United States, 2001-2010. Available from: https://www.cdc.gov/traumaticbraininjury/data/rates_ed_byage.html. [updated 2016; cited 2019]

6. Centers for Disease Control and Prevention. Ten leading causes of death and injury 2017 Available from: https://www.cdc.gov/injury/wisqars/ LeadingCauses.html. [updated 2019; cited 2019]

7. Kochanek PM, Tasker RC, Carney N, Totten AM, Adelson PD, Selden NR, et al. Guidelines for the management of pediatric severe traumatic brain injury,
Third Edition: Update of the Brain Trauma Foundation Guidelines, Executive Summary. Neurosurgery. 2019;84(6):1169-78 Available from: http://search. ebscohost.com/login.aspx?direct $=$ true $\& \mathrm{db}=\mathrm{cmedm} \& A N=30822776 \&$ site $=$ ehost-live\&scope=site. [cited 2019 Jun 25].

8. Gupta D, Sharma D, Kannan N, et al. Guideline adherence and outcomes in severe adult traumatic brain injury for the CHIRAG (Collaborative Head Injury and Guidelines) study. World Neurosurg. 2016;89:169-79 Available from: https://www.ncbi.nlm.nih.gov/pubmed/26806065.

9. Vavilala MS, Kernic MA, Wang J, Kannan N, Mink RB, Wainwright MS, Groner J, Bell MJ, Giza CC, Zatzick DF, Ellenbogen RG, Boyle LN, Mitchell PH, Rivara FP. Acute care clinical indicators associated with discharge outcomes in children with severe traumatic brain injury. Crit Care Med. 2014;42(10):225866 Available from: https://www.ncbi.n/m.nih.gov/pubmed/25083982.

10. Pineda JA, Leonard JR, Mazotas IG, et al. Effect of implementation of a paediatric neurocritical care programme on outcomes after severe traumatic brain injury: a retrospective cohort study. Lancet Neurol. 2013 12(1):45-52 Available from: https://www.ncbi.nlm.nih.gov/pubmed/232 00264.

11. Maas Al, Menon DK, Lingsma HF, Pineda JA, Sandel ME, Manley GT. Reorientation of clinical research in traumatic brain injury: report of an international workshop on comparative effectiveness research. J Neurotrauma. 2012;29(1):32-46.

12. English S, Turgeon A, Owen E, Doucette S, Pagliarello G, Mclntyre L. Protocol management of severe traumatic brain injury in intensive care units: a systematic review. Neurocritical Care. 2013;18(1):131-42 Available from: https://www.ncbi.nlm.nih.gov/pubmed/22890909. [cited 2019 Jun 25].

13. Gerber LM, Chiu YL, Carney N, Hartl R, Ghajar J. Marked reduction in mortality in patients with severe traumatic brain injury. J Neurosurg. 2013; 119(6):1583-90 Available from: https://www.ncbi.nlm.nih.gov/pubmed/24 098983.

14. Faul M, Wald MM, Rutland-Brown W, Sullivent EE, Sattin RW. Using a costbenefit analysis to estimate outcomes of a clinical treatment guideline: Testing the Brain Trauma Foundation guidelines for the treatment of severe traumatic brain injury. J Trauma. 2007;63(6):1271-8 Available from: https:// www.ncbi.nlm.nih.gov/pubmed/18212649.

15. Bennett TD, Statler KD, Korgenski EK, Bratton SL. Osmolar therapy in pediatric traumatic brain injury. Crit Care Med. 2012;40(1):208-15.

16. Bennett TD, Riva-Cambrin J, Keenan HT, Korgenski EK, Bratton SL. Variation in intracranial pressure monitoring and outcomes in pediatric traumatic brain injury. Arch Pediatr Adolesc Med. 2012;166(7):641-7.

17. Talving $P$, Karamanos $E$, Teixeira $P G$, et al. Intracranial pressure monitoring in severe head injury: compliance with Brain Trauma Foundation guidelines and effect on outcomes: a prospective study. J Neurosurg. 2013;119(5): 1248-54.

18. Adelson PD, Bratton SL, Carney NA, et al. Guidelines for the acute medical management of severe traumatic brain injury in infants, children, and adolescents. Chapter 1: introduction. Pediatr Crit Care Med. 2003:4(3 Suppl):S2-4.

19. Kochanek PM, Carney N, Adelson PD, et al. Guidelines for the acute medical management of severe traumatic brain injury in infants, children, and adolescents--second edition. Pediatr Crit Care Med. 2012;13(Suppl 1):S1-82.

20. Biersteker HA, Andriessen TM, Horn J, Franschman G, van der Naalt J, Hoedemaekers CW, Lingsma HF, Haitsma I, Vos PE. Factors influencing intracranial pressure monitoring guideline compliance and outcome after severe traumatic brain injury. Crit Care Med. 2012;40(6):1914-22 Available from: https://www.ncbi.nlm.nih.gov/pubmed/22488001.

21. Dean NP, Boslaugh S, Adelson PD, Pineda JA, Leonard JR. Physician agreement with evidence-based recommendations for the treatment of severe traumatic brain injury in children. J Neurosurg. 2007;107(5 Suppl): 387-91 Available from: https://www.ncbi.nlm.nih.gov/pubmed/18459901.

22. Traumatic brain injury: time to end the silence. Lancet Neurol. 2010;9(4):331. Available from: https://www.ncbi.nlm.nih.gov/pubmed/20298955

23. Van Cleve W, Kernic MA, Ellenbogen RG, Wang J, Zatzick DF, Bell MJ, Wainwright MS, Groner Jl, Mink RB, Giza CC, Boyle LN, Mitchell PH, Rivara FP, Vavilala MS, Project P. National variability in intracranial pressure monitoring and craniotomy for children with moderate to severe traumatic brain injury. Neurosurgery. 2013;73(5):746-52 Available from: https://www.ncbi.nlm.nih. gov/pubmed/23863766.

24. Grol R, Grimshaw J. From best evidence to best practice: effective implementation of change in patients' care. Lancet. 2003;362(9391):1225-30 Available from: https://www.ncbi.nlm.nih.gov/pubmed/14568747. 
25. Grol R. Successes and failures in the implementation of evidence-based guidelines for clinical practice. Med Care. 2001;39(8 suppl 2):|146-54 Available from: https:/www.ncbinlm.nih.gov/pubmed/11583121.

26. Haines $A$, Rogers $S$. Integrating research evidence into practice. In: Silagy $C$, Haines A, editors. Evidence-based practice in primary care. 2 London: BMJ Books; 2001. p. 157-74.

27. Oxman AD, Flottorp $S$. An overview of strategies to promote implementation of evidence-based health care. In: Silagy C, Haines A, editors. Evidence-based practice in primary care. 2nd ed. London: BMJ Books; 2001. p. 101-19.

28. Cabana MD, Rand CS, Powe NR, Wu AW, Wilson MH, Abboud PA, et al. Why don't physicians follow clinical practice guidelines? A framework for improvement. JAMA. 1999;282(15):1458-65 Available from: https://www. ncbi.nlm.nih.gov/pubmed/10535437.

29. Grol R. Personal paper. Beliefs and evidence in changing clinical practice BMJ. 1997;315(7105):418-21 Available from: https://www.ncbi.nlm.nih.gov/ pmc/articles/PMC2127297/.

30. Sorani MD, Morabito D, Rosenthal G, Giacomini KM, Manley GT. Characterizing the dose-response relationship between mannitol and intracranial pressure in traumatic brain injury patients using a highfrequency physiological data collection system. J Neurotrauma. 2008;25(4): 291-8 Available from: https://www.ncbi.nlm.nih.gov/pubmed/18373479.

31. Proctor $E$, Brownson R. Measurement issues in dissemination and implementation research. In: Brownson R, Colditz G, Proctor E, editors. Dissemination and implementation research in health. New York: Oxford University Press; 2012. p. 261-80.

32. Leonard M, Graham S, Bonacum D. The human factor: the critical importance of effective teamwork and communication in providing safe care. Qual Saf Health Care. 2004;13(Suppl 1):85-90 Available from: https:// www.ncbinlm.nih.gov/pubmed/15465961.

33. Bayley MT, Teasell RW, Wolfe DL, Gruen RL, Eng JJ, Ghajar J, Tavender E, Kua A, Bragge P. Where to build the bridge between evidence and practice? results of an international workshop to prioritize knowledge translation activities in traumatic brain injury care. J Head Trauma Rehabil. 2014;29(4): 268-76 Available from: https://www.ncbi.nlm.nih.gov/pubmed/24984091.

34. Glasgow RE, Phillips SM, Sanchez MA. Implementation science approaches for integrating eHealth research into practice and policy. Int J Med Inform. 2014;83(7):e1-11 Available from: https:/www.ncbi.nlm.nih.gov/pubmed/23 910896.

35. Bosch M, McKenzie JE, Mortimer D, Tavender EJ, Francis JJ, Brennan SE, Knott JC, Ponsford JL, Pearce A, OC DA, Grimshaw JM, Rosenfeld JV, Gruen RL, Green SE. Implementing evidence-based recommended practices for the management of patients with mild traumatic brain injuries in Australian emergency care departments: study protocol for a cluster randomised controlled trial. Trials. 2014;15(1):281 Available from: https://www.ncbi.nlm. nih.gov/pubmed/25012235.

36. Bickman L, Kelley SD, Athay M. The technology of measurement feedback systems. Couple Family Psychol. 2012;1(4):274-84.

37. Roshanov PS, Fernandes N, Wilczynski JM, et al. Features of effective computerised clinical decision support systems: meta-regression of 162 randomised trials. BMJ. 2013;346:f657.

38. Vinson C, Bickmore T, Farrell D, et al. Adapting research-tested computerized tailored interventions for broader dissemination and implementation. Transl Behav Med. 2011;1(1):93-102.

39. Kawamoto K, Houlihan CA, Balas EA, Lobach DF. Improving clinical practice using clinical decision support systems: a systematic review of trials to identify features critical to success. BMJ. 2005:330(7494):765

40. Stetler CB, Damschroder $L$, Helfrich CD, Hagedorn HJ. A guide for applying a revised version of the PARIHS framework for implementation. Implement Sci. 2011;6:99. https://doi.org/10.1186/1748-5908-6-99.

41. Kitson A, Harvey G, McCormack B. Enabling the implementation of evidence based practice: a conceptual framework. Qual Health Care. 1998;7(3):149-58 Available from: https://www.ncbi.nlm.nih.gov/pubmed/10185141.

42. Malhotra S, Jordan D, Shortliffe E, Patel VL. Workflow modeling in critical care: piecing together your own puzzle. J Biomed Inform. 2007;40(2):81-92.

43. Vennix JAM, Andersen DF, Richardson GP, Rohrbaugh J. Model-building for group decision support: issues and alternatives in knowledge elicitation. Eur J Oper Res. 1992:59(1):28-41 Available from: https://www.sciencedirect.com/ science/article/pii/037722179290005T.

44. Hovmand PS. Community based system dynamics. New York: Springer; 2014. p. xv-104.
45. Rouwette EAJA, Größler A, Vennix JAM. Exploring influencing factors on rationality: a literature review of dynamic decision-making studies in system dynamics. Syst Res Behav Sci. 2004;21(4):351-70. https://doi.org/ 10.1002/sres.647.

46. Andersen DF, Richardson GP, Vennix JAM. Group model building: adding more science to the craft. Syst Dyn Rev. 1997;13(2):187-201. https://doi.org/ 10.1002/(SICI) 1099-1727(199722)13:2<187:AID-SDR124>3.0.CO;2-O.

47. Landsverk J, Brown C, Chamberlain P, Palinkas L, Ogihara M, Czaja S, Goldhaber-Fiebert J, Rolls-Reutz J, Horwitz S. Design and analysis in dissemination and implementation research. In: Brownson R, Colditz G, Proctor $\mathrm{E}$, editors. Dissemination and implementation research in health. New York: Oxford University Press; 2012. p. 225-60.

48. Hovmand PS, Gillespie DF. Implementation of evidence-based practice and organizational performance. J Behav Health Serv Res. 2010;37(1):79-94.

49. Munar W, Hovmand PS, Fleming C, Darmstadt GL. Scaling-up impact in perinatology through systems science: bridging the collaboration and translational divides in cross-disciplinary research and public policy. Semin Perinatol. 2015;39(5):416-23.

50. Homa L, Rose J, Hovmand PS, et al. A participatory model of the paradox of primary care. Ann Fam Med. 2015;13(5):456-65.

51. Zhang Y, Padman R, Patel N. Paving the COWpath: learning and visualizing clinical pathways from electronic health record data. J Biomed Inform. 2015;58:186-97.

52. Chamberlain P, Brown CH, Saldana L. Observational measure of implementation progress in community based settings: the Stages of Implementation Completion (SIC). Implement Sci. 2011;6:116 Available from: https://www.ncbi.nlm.nih.gov/pubmed/21974914.

53. Krueger RA, Casey MA. Focus groups : a practical guide for applied research. 5th ed. Thousand Oaks: SAGE; 2015

54. Van Schaik PB-S, J.A.; Warren, J.G. Clinical acceptance of a low-cost portable system for postural assessment. Behav Inf Technol. 2002;21(1):47-57. doi: 10. 1080/01449290110107236.

55. Atkinson N. Developing a questionnaire to measure perceived attributes of eHealth innovations. Am J Health Behav. 2007;31(6):612-21 Available from: https://www.ncbi.nlm.nih.gov/pubmed/17691874.

56. Saldana $L$. The stages of implementation completion for evidencebased practice: protocol for a mixed methods study. Implement Sci. 2014;9(1):43 Available from: https://www.ncbi.nlm.nih.gov/pubmed/24 708893.

57. Palinkas LA, Aarons GA, Horwitz S, Chamberlain P. Hurlburt M, Landsverk J. Mixed method designs in implementation research. Adm Policy Ment Health. 2011;38(1):44-53 Available from: https://www.ncbi.nlm.nih.gov/ pubmed/20967495.

58. Rao AS, Adam TJ, Gensinger R, Westra BL. Study of the factors that promoted the implementation of electronic medical record on iPads at two emergency departments. AMIA Annu Symp Pro. 2012;2012:744-52 Available from: https://www.ncbi.nlm.nih.gov/pubmed/23304348.

59. R Core Team. A language and environment for statistical computing. Vienna: R Foundation for Statistical Computing; 2013. http://www.Rproject.org/

60. Lyon AR, Wasse JK, Ludwig K, Zachry M, Bruns EJ, Unützer J, McCauley E. The Contextualized Technology Adaptation Process (CTAP): Optimizing Health Information Technology to Improve Mental Health Systems. Adm Policy Ment Health. 2016;43(3):394-409. https://doi.org/10.1007/s10488-0150637-X.

61. Hsieh HF, Shannon SE. Three approaches to qualitative content analysis. Qual Health Res. 2005;15(9):1277-88.

62. Miyasaka KW, Martin ND, Pascual JL, Buchholz J, Aggarwal R. A simulation curriculum for management of trauma and surgical critical care patients. J Surg Educ. 2015;72(5):803-10.

63. Rabin B, Brownson R. Developing the terminology for dissemination and implementation research. In: Brownson R, Colditz G, Proctor E, editors. Dissemination and implementation research in health. New York: Oxford University Press; 2012. p. 23-51.

64. Stetler CB, Legro MW, Rycroft-Malone J, Bowman C, Curran G, Guihan M, Hagedorn $\mathrm{H}$, Pineros S, Wallace CM. Role of "external facilitation" in implementation of research findings: a qualitative evaluation of facilitation experiences in the veterans health administration. Implement Sci. 2006;1:23. https://doi.org/10.1186/1748-5908-1-23.

65. SISEP. Training Plan Template. 2013. http://implementation.fpg.unc.edu/ resources/training-plan-template. Accessed 1 Dec 2016. 
66. Rodriguez BJ, Loman SL, Horner RH. A preliminary analysis of the effects of coaching feedback on teacher implementation fidelity of first step to success. Behav Anal Pract. 2009;2(2):11-21.

67. Galukande M, Kaggwa S, Sekimpi P, et al. Use of surgical task shifting to scale up essential surgical services: a feasibility analysis at facility level in Uganda. BMC Health Serv Res. 2013;13:292.

68. Bowen DJ, Kreuter M, Spring B, et al. How we design feasibility studies. Am J Prev Med. 2009;36(5):452-7.

69. Martens JD, van der Weijden T, Winkens RA, et al. Feasibility and acceptability of a computerised system with automated reminders for prescribing behaviour in primary care. Int J Med Inform. 2008;77(3):199-207.

70. Beatty PC, Willis GB. Research synthesis: the practice of cognitive interviewing. Public Opinion Q. 2007;71(2):287-311.

71. Tasker RC, Menon DK. Critical care and the brain. JAMA. 2016;315(8):749-50.

\section{Publisher's Note}

Springer Nature remains neutral with regard to jurisdictional claims in published maps and institutional affiliations.

Ready to submit your research? Choose BMC and benefit from:

- fast, convenient online submission

- thorough peer review by experienced researchers in your field

- rapid publication on acceptance

- support for research data, including large and complex data types

- gold Open Access which fosters wider collaboration and increased citations

- maximum visibility for your research: over $100 \mathrm{M}$ website views per year

At BMC, research is always in progress.

Learn more biomedcentral.com/submissions 